УДК 531. 383-11: 681.7

\author{
С.Г. Николаев, Ю.В. Ившина
}

Пермский национальный исследовательский политехнический университет, Пермь, Россия

ИДЕНТИФИКАЦИЯ ИНСТРУМЕНТАЛЬНЫХ ПОГРЕШНОСТЕЙ ВОЛОКОННО-ОПТИЧЕСКИХ ГИРОСКОПОВ БЕСПЛАТФОРМЕННОЙ ИНЕРЦИАЛЬНОЙ НАВИГАЦИОННОЙ СИСТЕМЫ

\begin{abstract}
Рассматривается проблема повышения точности идентификации инструментальных погрешностей волоконно-оптических гироскопов (ВОГ) бесплатформенных инерциальных навигационных систем (БИНС). Погрешности ВОГ определяются на имитаторе движения в процессе калибровки БИНС. С помощью математического моделирования исследуется метод определения инструментальных погрешностей волоконно-оптических гироскопов по ошибкам выходных сигналов БИНС. Компоненты вектора ошибок выходных сигналов измеряются при калибровке БИНС. С другой стороны, вектор ошибок есть решение модели ошибок БИНС. Входными сигналами модели ошибок БИНС являются инструментальные погрешности волоконно-оптических гироскопов. Метод базируется на решении обратной задачи - определении инструментальных погрешностей волоконно-оптических гироскопов по ошибкам выходным сигналов БИНС, определенным экспериментально в процессе калибровки. Целями моделирования являются: проверка возможности реализации предлагаемого способа, анализ его эффективности, апробация алгоритмов идентисрикации и исследование влияния шумов волоконно-оптических гироскопов на точность определения их инструментальных погрешностей. Моделирование осуществляется в среде MATLAB.
\end{abstract}

Ключевые слова: волоконно-оптический гироскоп, инструментальные погрешности, бесплатформенная инерциальная навигационная система, калибровка, модель ошибок.

\author{
S.G. Nikolaev, I.V. Ivshina
}

Perm National Research Polytechnic University, Perm, Russian Federation

\title{
IDENTIFICATION OF INSTRUMENTAL ERRORS OF FIBER-OPTIC GYROSCOPES OF STRAPDOWN INERTIAL NAVIGATION SYSTEM
}

The problem of increasing the accuracy of the identification of instrumental errors of fiber-optic gyroscopes of strapdown inertial navigation systems (SINS) is considered. Error identification of fiberoptic gyroscopes is run on the motion simulator during calibration of SINS. Method for the determination of instrumental errors of fiber-optic gyroscopes using errors of the output signals of the SINS is investigated using mathematical modeling. Components of the error vector of output signals are measured during the calibration of SINS. Else the error vector is the solution of the model errors of SINS. The 
input signals of the model errors of SINS are instrumental error of fiber-optic gyroscopes. The method is based on solving the inverse problem, it is the determination of the instrumental errors of fiber-optic gyroscopes using errors of output signal of SINS, errors of output signal was defined experimentally in the calibration process. The purpose of the simulation is: validating the feasibility of the proposed method, the analysis of its efficiency, testing of algorithms for the identification and study of the influence of noise fiber-optic gyroscopes on the accuracy of their instrumental errors. The simulation is done using MATLAB.

Keywords: fiber-optic gyroscope, instrumental error, strapdown inertial navigation system, calibration, error model.

Качество идентификации систематических инструментальных погрешностей инерциальных измерителей (волоконно-оптических гироскопов и акселерометров) является определяющим требованием для бесплатформенных инерциальных навигационных систем (БИНС) навигационного класса. Процесс определения инструментальных погрешностей инерциальных измерителей называется калибровкой БИНС.

Калибровка выполняется на имитаторе движения (наклонно-поворотном калибровочном стенде). Стенд ориентируется относительно плоскостей горизонта и меридиана, может разворачиваться на постоянные углы и вращаться. Угловые скорости вращения и углы имитатора измеряются с высокой точностью. Суть калибровки заключается в определении реакции инерциального измерителя на действие калибровочного (эталонного) сигнала. В качестве калибровочных сигналов для акселерометров и гироскопов используется ускорение силы тяжести $g$, угловая скорость суточного вращения Земли и угловые скорости стенда.

Вычисленные в процессе калибровки инструментальные погрешности волоконно-оптических гироскопов и акселерометров называются калибровочными коэффициентами. После окончания калибровки они «зашиваются» в бортовой вычислитель БИНС для компенсации систематических погрешностей инерциальных измерителей. Основным недостатком традиционного способа калибровки является высокий уровень шумов в сигналах инерциальных измерителей.

Разрешение этого противоречия возможно с помощью калибровки по значительно менее зашумленным ошибкам выходных сигналов БИНС $[1,2]$. При данном способе калибровка проводится в два этапа. На первом этапе решается обратная задача определения входных сигналов модели ошибок БИНС, которые являются функциями систематических ошибок акселерометров и гироскопов. На втором этапе по полученным входным сигналам модели ошибок определяются калибровочные коэффициенты. 


\section{1. Постановка задачи и цели моделирования}

Необходимо разработать математическую модель процесса калибровки бесплатформенной инерциальной навигационной системы по ошибкам ее выходных сигналов. Целями моделирования являются: проверка реализации предлагаемого способа идентификации инструментальных погрешностей волоконно-оптических гироскопов, анализ его эффективности, апробация алгоритмов идентификации и исследование влияния шумов волоконно-оптических гироскопов на точность определения их инструментальных погрешностей.

\section{2. Математическая модель идентификации инструментальных погрешностей волоконно-оптических гироскопов}

В процессе идентификации погрешностей ВОГ будем считать, что инструментальные погрешности акселерометров равны нулю. Весь технологический процесс калибровки БИНС можно представить в виде структуры, содержащей две части. В первой части проводятся измерения калибровочных сигналов инерциальными измерителями в различных ориентациях БИНС. Во второй части для каждой ориентации: моделируется работа БИНС в автономном режиме; вычисляется вектор ошибок выходных сигналов БИНС; вычисляется производная вектора ошибок выходных сигналов. Идентификация инструментальных погрешностей волоконно-оптических гироскопов выполняется во второй части в режиме постобработки в два этапа. На первом этапе вычисляются входные сигналы модели ошибок БИНС. На втором этапе определяются инструментальные погрешности волоконно-оптических гироскопов.

Математическая модель идентификации инструментальных погрешностей волоконно-оптических гироскопов представлена на рис. 1. Модель содержит восемь модулей. Рассмотрим назначение и свойства каждого модуля. Первая часть структуры технологического процесса калибровки включает два модуля: модуль ИИБ (инерциально-измерительный блок) и модуль ИД (имитатор движения).

Модуль ИД моделирует работу имитатора движения - задает требуемые ориентации БИНС (связанной системы координат $O X Y Z$ ) относительно навигационного трехгранника OnUpe. Ориентация осей OnUpe: $O n$ лежит в плоскости горизонта и направлена на север, $O U p$ направлена по местной вертикали, Oе лежит в плоскости горизонта и направлена на 


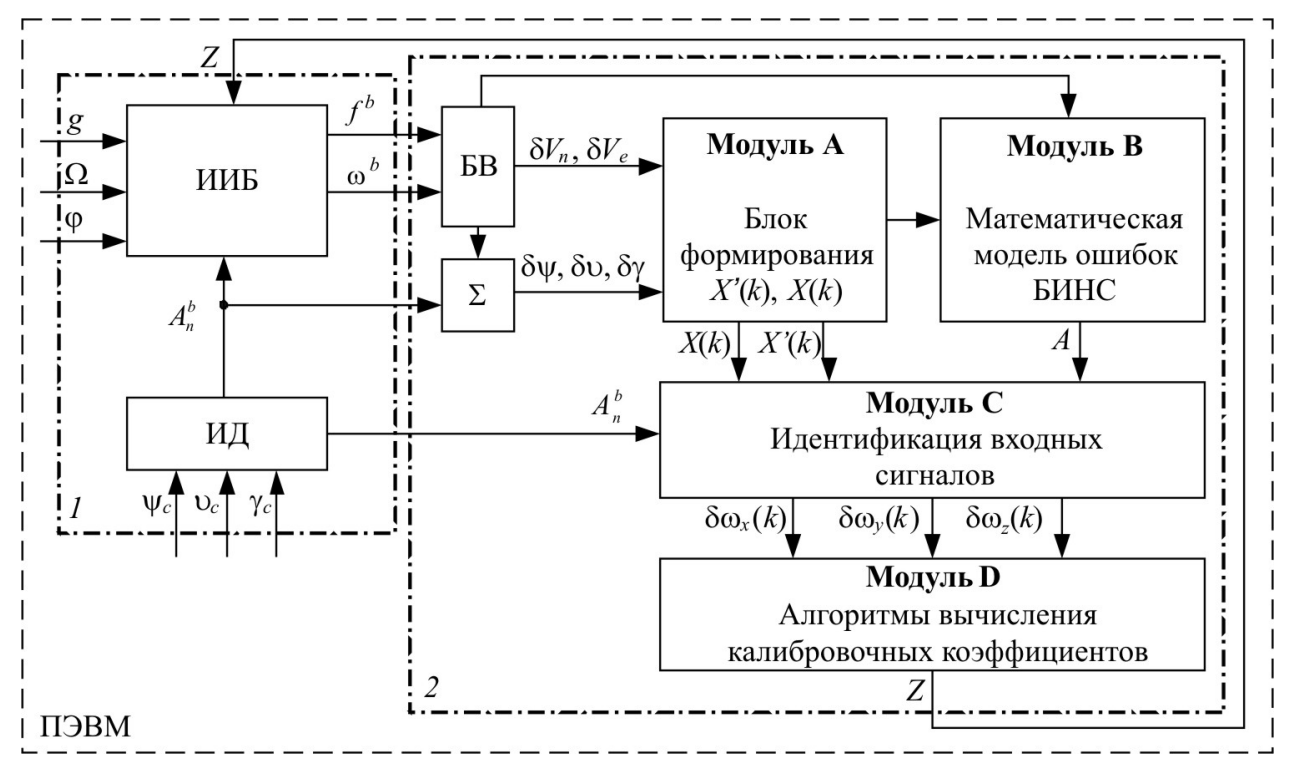

Рис. 1. Структурная схема математической модели идентификации инструментальных ошибок ВОГ БИНС

восток. ИД для каждой ориентации задает требуемые углы разворота $\psi_{c}$, $v_{c}, \gamma_{c}$ по курсу, тангажу и крену. Этим углам соответствует матрица $A_{n}^{b}$ перехода от навигационного трехгранника OnUpe к связанной системе координат $O X Y Z$.

Модуль ИИБ моделирует работу инерциально-измерительного блока БИНС. Сигналы волоконно-оптических гироскопов и акселерометров являются выходными сигналами ИИБ. Структуру моделей инструментальных ошибок волоконно-оптических гироскопов примем в стандартном виде. Погрешности ВОГ включают смещения нулей, ошибки масштабных коэффициентов, углы перекосов осей чувствительности и непараметрические флуктуационные погрешности. Математическая модель ИИБ - это система матричных уравнений, определяющих значения выходных сигналов инерциальных измерителей:

$$
\left(\begin{array}{l}
f_{x} \\
f_{y} \\
f_{z}
\end{array}\right)=A_{n}^{b}\left(\begin{array}{c}
0 \\
-g \\
0
\end{array}\right) ; \quad\left(\begin{array}{l}
\omega_{x} \\
\omega_{y} \\
\omega_{z}
\end{array}\right)=\left(\begin{array}{l}
\omega_{k x} \\
\omega_{k y} \\
\omega_{k z}
\end{array}\right)+\left(\begin{array}{c}
\delta \omega_{x} \\
\delta \omega_{y} \\
\delta \omega_{z}
\end{array}\right)
$$




$$
\begin{aligned}
& \left(\begin{array}{l}
\delta \omega_{x} \\
\delta \omega_{y} \\
\delta \omega_{z}
\end{array}\right)=\left(\begin{array}{l}
\omega_{n x} \\
\omega_{n y} \\
\omega_{n z}
\end{array}\right)+N_{d} \cdot\left(\begin{array}{l}
\omega_{k x} \\
\omega_{k y} \\
\omega_{k z}
\end{array}\right)+m_{d} ; \\
& N_{d}=\left(\begin{array}{ccc}
\delta k_{d x} & v_{x y} & -v_{x z} \\
-v_{y x} & \delta k_{d y} & v_{y z} \\
v_{z x} & -v_{z y} & \delta k_{d z}
\end{array}\right),
\end{aligned}
$$

где $f_{i}, i=x, y, z$ - сигналы акселерометров; $g$ - ускорение силы тяжести; $\omega_{i}, i=x, y, z$ - сигналы волоконно-оптических гироскопов; $\omega_{n i}, i=x, y$, $z$ - смещения нулей гироскопов; $\delta k_{d i}, i=x, y, z$ - ошибки масштабных коэффициентов гироскопов; $v_{i j}, i \neq j$ - углы перекосов осей чувствительности гироскопов; $m_{d}-$ непараметрические флуктуационные погрешности гироскопов; $\omega_{k i}, i=x, y, z-$ проекции калибровочных сигналов на оси чувствительности гироскопов; $A_{n}^{b}$ - матрица перехода от навигационного трехгранника $O n U p e$ к связанной системе координат $O X Y Z$.

Модуль БВ моделирует работу программно-математического обеспечения бортового вычислителя БИНС. Математическая модель БВ - программа, выполняющая решение основного векторного уравнения инерциальной навигации и матричного кинематического уравнения Пуассона, записанных для принятых выше условий.

$$
\begin{gathered}
\left\{\begin{array}{c}
\delta \dot{V}=\tilde{A}_{b}^{n} f^{b}-2 u_{i e}^{n} \cdot \delta V+g \\
\dot{A}_{b}^{n}=A_{b}^{n} \hat{\omega}_{b}^{b}-\hat{u}_{n}^{n} A_{b}^{n}
\end{array} ;\right. \\
u_{i e}^{n}=\left(\begin{array}{c}
\Omega \cos \varphi \\
\Omega \sin \varphi \\
0
\end{array}\right) ; \quad \hat{\omega}_{b}^{b}=\left(\begin{array}{ccc}
0 & \omega_{z} & -\omega_{y} \\
-\omega_{z} & 0 & \omega_{x} \\
\omega_{y} & -\omega_{x} & 0
\end{array}\right) ; \\
\hat{u}_{n}^{n}=\left(\begin{array}{ccc}
\delta V n & -\Omega \sin \varphi-\frac{\delta V e}{a} \operatorname{tg} \varphi \\
-\frac{\delta V n}{a} & 0 & \Omega \cos \varphi+\frac{\delta V e}{a} \\
\Omega \sin \varphi+\frac{\delta V e}{a} \operatorname{tg} \varphi & -\Omega \cos \varphi-\frac{\delta V e}{a} & 0
\end{array}\right),
\end{gathered}
$$


где $\delta V$ - вектор ошибок вычисления линейной скорости объекта; $\Omega-$ угловая скорость суточного вращения Земли; $g$ - ускорение силы тяжести; $a$ - радиус Земли; $\varphi$ - широта; $\tilde{A}_{b}^{n}$ - вычисленная матрица $A_{b}^{n}$.

В модуле $\sum$ вычисляются ошибки углов рыскания, тангажа и крена и формируется кососимметрическая матрица $\chi$, элементами которой являются указанные выше ошибки:

$$
\chi=\left(\begin{array}{ccc}
0 & \delta \vartheta & -\delta \psi \\
-\delta \vartheta & 0 & \delta \gamma \\
\delta \psi & -\delta \gamma & 0
\end{array}\right),
$$

где $\delta \psi, \delta \vartheta, \delta \gamma-$ ошибки углов рысканья, тангажа и крена.

В модуле $A$ формируется вектор ошибок выходных сигналов БИНС $X(k)$ и вычисляется его производная $\dot{X}(k)$. Для снижения влияния шумовой составляющей перед численным дифференцированием вектор ошибок аппроксимируется полиномом.

В модуле $B$ в соответствии с алгоритмами бортового вычислителя БВ формируется модель ошибок БИНС [3]:

$$
A=\left(\begin{array}{ccccccc}
\dot{X}=A X+F ; \\
\frac{1}{a} & 0 & 0 & - \text { Uup } & -\frac{U u p}{a} & 0 & 0 \\
-2 U u p & 0 & 0 & g & 0 & 0 & 0 \\
0 & U u p & -\frac{1}{a} & 0 & 0 & -\frac{U u p}{a} & -U n \\
0 & 0 & 1 & 0 & 0 & 0 & 0 \\
1 & 0 & 0 & 0 & 0 & 0 & 0 \\
\frac{\operatorname{tg} \varphi}{a} & 0 & 0 & U n & \frac{U n}{a} & 0 & 0
\end{array}\right) ;
$$

где $\delta V e, \delta V n-$ ошибки восточной и северной составляющих линейной скорости объекта; $\delta r n, \delta r e-$ ошибки координат в северном и восточном направлениях; $U n=\Omega \cos \varphi ; U u p=\Omega \sin \varphi$. 
В модуле $C$ выполняется первый этап идентификации инструментальных погрешностей ВОГ, в котором определяется вектор входных сигналов модели ошибок бесплатформенной инерциальной навигационной системы $F(k)$ для каждой ориентации БИНС, задаваемой ИД. По вектору $F(k)$ вычисляются инструментальные ошибки ВОГ. В этом случае инструментальные ошибки ВОГ определяются не через зашумленные сигналы гироскопов, а с помощью малошумных ошибок выходных сигналов БИНС. Величины инструментальных ошибок ВОГ зависят от значений калибровочных коэффициентов и калибровочных сигналов. Общее число калибровочных коэффициентов трех ВОГ БИНС равно 12 (3 ошибки масштабных коэффициентов, 3 смещения нуля и 6 углов перекосов, по 2 угла на ось чувствительности каждого ВОГ). Для формирования калибровочных уравнений, с помощью которых определяются калибровочные коэффициенты, необходимо не менее 12 ориентаций БИНС. Выберем в качестве плоскости калибровки, в которой будут располагаться оси чувствительности ВОГ, плоскость меридиана $n U p$. С этой плоскостью будем последовательно совмещать плоскости осей чувствительности ВОГ $x y$ и $y z$. Оси чувствительности волоконно-оптических гироскопов будем располагать под углом $45^{\circ}$ к осям $O n$ и $O U p$. При совмещении плоскости $x y$ с плоскостью меридиана после 4 последовательных разворотов осей $x$ и $y$ вокруг оси $O e$ с шагом $45^{\circ}$ получим 4 ориентации осей $x$ и $y$. После выполнения аналогичных операций с осями $x, y$ и осями $y, z$ получим 12 ориентаций осей чувствительности ВОГ.

Для каждой ориентации формируется вектор ошибок выходных сигналов $X(k)$ и вычисляется его производная $\dot{X}(k)$. Вектор входных сигналов $F(k)$ модели ошибок БИНС и инструментальные ошибки ВОГ определяются с помощью зависимостей:

$$
\begin{gathered}
F(k)=\dot{X}(k)-A \cdot X(k) ; \\
F(k)=\left(0, \delta \omega_{n}(k), 0, \delta \omega_{e}(k), 0,0, \delta \omega_{u p}(k)\right)^{T} ; \\
\left(\begin{array}{l}
\delta \omega_{x} \\
\delta \omega_{y} \\
\delta \omega_{z}
\end{array}\right)=A_{n}^{b}\left(\begin{array}{c}
\delta \omega_{n} \\
\delta \omega_{u p} \\
\delta \omega_{e}
\end{array}\right) .
\end{gathered}
$$


Матрица $A$ имеет одно собственное значение, равное нулю. Это приводит к неустойчивости модели ошибок по углу рыскания и вызывает существенные погрешности определения вектора $F(k)$. Проблему можно решить с помощью введения корректирующего сигнала от калибровочного стенда. Если в алгоритмах БИНС в каждый расчетный

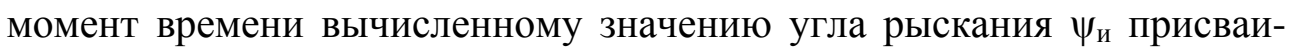
вать значение угла рыскания $\psi_{c}$, заданного ИД, то можно осуществить редукцию модели ошибок навигационной системы, уменьшив ее порядок на единицу. Рассмотрим способ такой коррекции более подробно.

В этом случае модель ошибок БИНС будет иметь вид

$$
\dot{X}_{1}=A_{1} \cdot X_{1}+F_{1}
$$

где $A_{1}$ - матрица, полученная из матрицы $A$ вычеркиванием последней строки и последнего столбца; $X_{1}=\left(\delta V_{e}, \delta \alpha_{n}, \delta V_{n}, \alpha_{e}, \delta r_{n}, \delta r_{e}\right)^{T}$, $F_{1}=\left(0, \delta \omega_{n}, 0, \delta \omega_{e}, 0,0\right)^{T}$. Матрица $A_{1}$ не имеет собственных нулевых значений. Модель ошибок БИНС устойчивая. В этом случае определяются составляющие вектора входных сигналов $\delta \omega_{n}$ и $\delta \omega_{e}$, а составляющую $\delta \omega_{u p}$ вычисляем по выходным сигналам ВОГ.

В модуле $D$ вычисляется вектор калибровочных коэффициентов волоконно-оптических гироскопов $Z$. Вектор $Z$ имеет размерность, равную 12 по числу калибровочных коэффициентов. Составляющими вектора $Z$ являются 3 ошибки масштабных коэффициентов $\delta k_{d i}, 3$ смещения нуля $\omega_{n i}, 6$ углов перекосов осей чувствительности ВОГ $v_{i j}$. Отличие от традиционной методики вычислений калибровочных коэффициентов заключается в том, что вместо показаний ВОГ используются вычисленные ошибки инструментальных погрешностей $\delta \omega_{i}(k)$ волоконно-оптических гироскопов. Для определения калибровочных коэффициентов ВОГ применим методику скалярного способа калибровки. Калибровочные соотношения скалярного способа получаются с помощью скалярного произведения векторов калибровочного сигнала и показаний датчиков. В нашем случае будем использовать скалярное произведение векторов калибровочных сигналов и векторов инструментальных погрешностей ВОГ.

Перейдем к безразмерным моделям ошибок волоконно-оптических гироскопов [4]. Для этого необходимо разделить левые и правые части уравнений (1) на скаляр $\Omega_{0}$, являющийся модулем вектора угловой скорости суточного вращения Земли: 


$$
\frac{\delta \omega_{i}(k)}{\Omega_{0}}=N_{d} \cdot n_{d}(k)+\Omega_{0}^{-1} \cdot C_{a}+\Omega_{0}^{-1} \cdot m_{d}(k),
$$

где $k$ - номер ориентации, $\omega_{k i}(k)$ - калибровочные сигналы;

$$
n_{d}(k)=\Omega_{0}^{-1}\left(\begin{array}{c}
\omega_{k x}(k) \\
\omega_{k y}(k) \\
\omega_{k z}(k)
\end{array}\right), \quad C_{d}=\left(\begin{array}{c}
\omega_{n x} \\
\omega_{n y} \\
\omega_{n z}
\end{array}\right) .
$$

Ввиду того, что в каждой ориентации ИД проводились процедуры осреднения показаний волоконно-оптических гироскопов, безразмерным вектором шумов $\Omega_{0}{ }^{-1} \cdot m_{d}(k)$ будем пренебрегать.

Основные калибровочные соотношения для вычисления калибровочных коэффициентов получим, применив к векторной зависимости (7) операцию скалярного умножения вектора относительного калибровочного сигнала и вектора относительных погрешностей ВОГ [4]:

$$
n_{d}^{T}(k) \cdot \frac{\delta \omega_{i}(k)}{\Omega_{0}}=n_{d}^{T}(k) \cdot N_{d} \cdot n_{d}(k)+\Omega_{0}^{-1} \cdot n_{d}^{T}(k) \cdot C_{d},
$$

где $n_{d}(k)=f_{k}\left(n_{d 1}, n_{d 2}\right) ; n_{d 1}=\cos \varphi ; n_{d 2}=\sin \varphi$.

Для одной ориентации ИД по скалярному уравнению (8) получаем одну зависимость для определения калибровочных коэффициентов волоконно-оптических гироскопов.

Обозначим значение скалярного произведения вектора калибровочного сигнала и вектора инструментальных погрешностей ВОГ в ориентации $k$ :

$$
S_{d}(k)=n_{d}^{T}(k) \frac{\delta \omega_{i}(k)}{\Omega_{0}}
$$

Для ориентаций $k=1 \ldots 12$ скалярные произведения $S_{d}(k)$, выраженные через калибровочные коэффициенты ВОГ, имеют вид:

а) для $k=1 . . .4$

$$
\begin{aligned}
& S_{d}(k)=n_{d x}^{2} \delta k_{d x}+n_{d y}^{2} \delta k_{d y}+n_{d x} n_{d y}\left(v_{x y}-v_{y x}\right)+n_{d x} \cdot \frac{\omega_{n x}}{\Omega_{0}}+n_{d y} \cdot \frac{\omega_{n y}}{\Omega_{0}}, \\
& n_{d x}=f_{k}\left(n_{d 1}, n_{d 2}\right) ; \quad n_{d y}=f_{k}\left(n_{d 1}, n_{d 2}\right) ;
\end{aligned}
$$


б) для $k=5 \ldots 8$

$$
\begin{aligned}
& S_{d}(k)=n_{d y}^{2} \delta k_{d y}+n_{d z}^{2} \delta k_{d z}+n_{d y} n_{d z}\left(v_{y z}-v_{z y}\right)+n_{d y} \cdot \frac{\omega_{n y}}{\Omega_{0}}+n_{d z} \cdot \frac{\omega_{n z}}{\Omega_{0}}, \\
& n_{d y}=f_{k}\left(n_{d 1}, n_{d 2}\right) ; \quad n_{d z}=f_{k}\left(n_{d 1}, n_{d 2}\right) ;
\end{aligned}
$$

в) для $k=9 \ldots 12$

$$
\begin{aligned}
& S_{d}(k)=n_{d x}^{2} \delta k_{d x}+n_{d z}^{2} \delta k_{d z}+n_{d x} n_{d z}\left(v_{z x}-v_{x z}\right)+n_{d x} \cdot \frac{\omega_{n x}}{\Omega_{0}}+n_{d z} \cdot \frac{\omega_{n z}}{\Omega_{0}}, \\
& n_{d x}=f_{k}\left(n_{d 1}, n_{d 2}\right) ; \quad n_{d z}=f_{k}\left(n_{d 1}, n_{d 2}\right) .
\end{aligned}
$$

Используя калибровочные соотношения (9), получим выражения для вычисления калибровочных коэффициентов:

$$
\begin{aligned}
& \delta k_{d x}=\frac{1}{4}\left(\sum_{1-4} S_{d}(k)-\sum_{5-8} S_{d}(k)+\sum_{9-12} S_{d}(k)\right) \\
& \delta k_{d y}=\frac{1}{4}\left(\sum_{1-4} S_{d}(k)+\sum_{5-8} S_{d}(k)-\sum_{9-12} S_{d}(k)\right) \text {; } \\
& \delta k_{d z}=\frac{1}{4}\left(-\sum_{1-4} S_{d}(k)+\sum_{5-8} S_{d}(k)+\sum_{9-12} S_{d}(k)\right) \text {; } \\
& \frac{\omega_{n x}}{\Omega_{0}}=-\frac{1}{2 \sqrt{2}\left(n_{d_{1}}+n_{d_{2}}\right)}\left(\frac{n_{d_{1}}}{n_{d_{2}}}\left(\sum_{3,4,5,6} S_{d}(k)-\sum_{1,2,7,8} S_{d}(k)\right)+\sum_{11,12} S_{d}(k)-\sum_{9,10} S_{d}(k)\right) \\
& \frac{\omega_{n y}}{\Omega_{0}}=-\frac{1}{2 \sqrt{2}\left(n_{d_{1}}+n_{d_{2}}\right)}\left(\frac{n_{d_{2}}}{n_{d_{1}}}\left(\sum_{1,2,11,12} S_{d}(k)-\sum_{3,4,9,10} S_{d}(k)\right)+\sum_{5,6} S_{d}(k)-\sum_{7,8} S_{d}(k)\right) \text {; } \\
& \frac{\omega_{n z}}{\Omega_{0}}=-\frac{1}{2 \sqrt{2}\left(n_{d_{1}}+n_{d_{2}}\right)}\left(\sum_{1,2,7,8,11,12} S_{d}(k)-\sum_{3,4,5,6,9,10} S_{d}(k)\right) \text {; } \\
& \Delta_{d y}=v_{x y}-v_{y x}=\frac{1}{2\left(n_{d_{1}}^{2}-n_{d_{2}}^{2}\right)}\left(\sum_{2,4} S_{d}(k)-\sum_{1,3} S_{d}(k)+2 n_{d_{1}} n_{d_{2}}\left(\sum_{9-12} S_{d}(k)-\sum_{5-8} S_{d}(k)\right)\right) ; \\
& \Delta_{d y z}=v_{y z}-v_{z y}=\frac{1}{2\left(n_{d_{1}}^{2}-n_{d_{2}}^{2}\right)}\left(\sum_{6,8} S_{d}(k)-\sum_{5,7} S_{d}(k)-2 n_{d_{1}} n_{d_{2}}\left(\sum_{1-4} S_{d}(k)-\sum_{9-12} S_{d}(k)\right)\right) \text {; } \\
& \Delta_{d x z}=v_{x z}-v_{z x}=\frac{1}{2\left(n_{d_{1}}^{2}-n_{d_{2}}^{2}\right)}\left(\sum_{10,12} S_{d}(k)-\sum_{9,11} S_{d}(k)+2 n_{d_{1}} n_{d_{2}}\left(\sum_{1-4} S_{d}(k)-\sum_{5-8} S_{d}(k)\right)\right) \text {. }
\end{aligned}
$$


Основные связи между модулями модели идентификации инструментальных погрешностей ВОГ показаны на структурной схеме модели, представленной на рис. 1. Рассматриваемая модель позволяет построить итерационный процесс для повышения точности определения погрешностей. Вычисленный вектор калибровочных коэффициентов $Z$ вводится в качестве обратной связи в БВ БИНС, и процесс идентификации повторяется. Собственно моделирование выполняется во второй части структуры технологического процесса калибровки отдельно для каждой ориентации БИНС. Время моделирования делится на два временных интервала: время выставки БИНС и время работы БИНС в автономном режиме. Затем во второй части структуры технологического процесса калибровки в режиме постобработки выполняются расчеты значений входных сигналов модели ошибок БИНС и калибровочных коэффициентов.

\section{3. Верификация модели}

Верификация математической модели идентификации инструментальных погрешностей волоконно-оптических гироскопов бесплатформенной инерциальной навигационной системы выполнялась методом пробного прогона модели.

Исходные параметры для моделирования задаются в модулях ИД, ИИБ и БВ. Были взяты следующие параметры модулей модели.

Модуль ИД: ориентации $k=1 . .4$, плоскость $x y$ системы $O X Y Z$ совпадает с плоскостью меридиана $n U p$, угол разворота ИД равен 45, 135, 225,315 ; ориентации $k=5 \ldots 8$, плоскость $y z$ системы $O X Y Z$ совпадает с плоскостью меридиана $n U p$, угол разворота ИД равен $45,135,225,315$; ориентации $k=9 \ldots 12$, плоскость х $z$ системы $O X Y Z$ совпадает с плоскостью меридиана $n U p$, угол разворота ИД равен 45, 135, 225, 315.

Модуль ИИБ: выходные сигналы акселерометров идеальные, выходные сигналы ВОГ не содержат шумовых составляющих:

$$
\begin{aligned}
\left(\begin{array}{l}
f_{x} \\
f_{y} \\
f_{z}
\end{array}\right)=A_{n}^{b}\left(\begin{array}{c}
0 \\
-g \\
0
\end{array}\right) ; \quad\left(\begin{array}{l}
\omega_{x} \\
\omega_{y} \\
\omega_{z}
\end{array}\right)=\left(\begin{array}{l}
\omega_{k x} \\
\omega_{k y} \\
\omega_{k z}
\end{array}\right)+\left(\begin{array}{l}
\delta \omega_{x} \\
\delta \omega_{y} \\
\delta \omega_{z}
\end{array}\right) ; \\
\left(\begin{array}{l}
\delta \omega_{x} \\
\delta \omega_{y} \\
\delta \omega_{z}
\end{array}\right)=\left(\begin{array}{l}
\omega_{n x} \\
\omega_{n y} \\
\omega_{n z}
\end{array}\right)+N_{d} \cdot\left(\begin{array}{l}
\omega_{k x} \\
\omega_{k y} \\
\omega_{k z}
\end{array}\right) ;
\end{aligned}
$$




$$
\begin{gathered}
N_{d}=\left(\begin{array}{ccc}
\delta k_{d x} & v_{x y} & -v_{x z} \\
-v_{y x} & \delta k_{d y} & v_{y z} \\
v_{z x} & -v_{z y} & \delta k_{d z}
\end{array}\right) ; \\
\delta k_{d x}=0,02297554 ; \quad \delta k_{d y}=0,0229639 ; \quad \delta k_{d z}=0,02293191 ; \\
\omega_{n x}=0,0124 \text { град/ч; } \quad \omega_{n y}=-0,0041 \text { град/ч; } \quad \omega_{n z}=0,0351 \text { град/ч; } \\
v_{x y}=-0,00025^{\circ} ; \quad v_{y x}=0,0145^{\circ} ; \quad v_{z x}=0,0145^{\circ} ; \\
v_{x z}=-0,0426^{\circ} ; \quad v_{y z}=-0,0142^{\circ} ; \quad v_{z y}=-0,0142^{\circ} .
\end{gathered}
$$

\section{Модуль БВ:}

Время работы БИНС в автономном режиме в каждой ориентации было принято равным 10 мин.

$$
\begin{array}{cc}
\Omega=15,041 \text { град } / \mathrm{q} ; & \varphi=58^{\circ} ; \\
a=6378137 \mathrm{M} ; & g=9,8211972983 \mathrm{M} / \mathrm{c} .
\end{array}
$$

Полученные в процессе моделирования относительные погрешности вычисленных значений калибровочных коэффициентов волоконнооптических гироскопов находятся в диапазоне $\pm\left(1,51 \cdot 10^{-14} \ldots 4,8 \cdot 10^{-10}\right) \%$, что соответствует уровню ошибок вычисления и подтверждает адекватность принятой модели идентификации.

\section{4. Численный эксперимент}

Проведем численный эксперимент идентификации инструментальных погрешностей волоконно-оптических гироскопов БИНС при наличии шумов в сигналах ВОГ. Сигналы акселерометров и ВОГ зададим по зависимостям (1).

Значения составляющих вектора калибровочных коэффициентов ВОГ зададим равными составляющим вектора, принятым при верификации модели.

Уровень шума $m_{d}$ будем оценивать средним квадратическим отклонением (СКО) случайной составляющей угловой скорости дрейфа ВОГ. Диапазон изменения СКО дрейфа ВОГ примем равным в пределах диапазона $\pm\left(1 \cdot 10^{-8} \ldots 1 \cdot 10^{-6}\right)$ рад/с. Уровень случайного дрейфа гироскопов навигационного класса лежит в пределах 0,001-0,02 град/ч, что соответствует уровню $\pm\left(1 \cdot 10^{-8} \ldots 1 \cdot 10^{-7}\right)$ рад/с. Относительные погрешности компонент вектора калибровочных коэффициентов ВОГ, вычисленные в процессе моделирования, представлены на рис. 2-4 в виде графиков зависимостей $\delta\left(\delta k_{d i}\right)=f\left(m_{d}\right), \delta\left(\omega_{n i}\right)=f\left(m_{d}\right)$ и $\delta\left(v_{i j}\right)=f\left(m_{d}\right)$. 


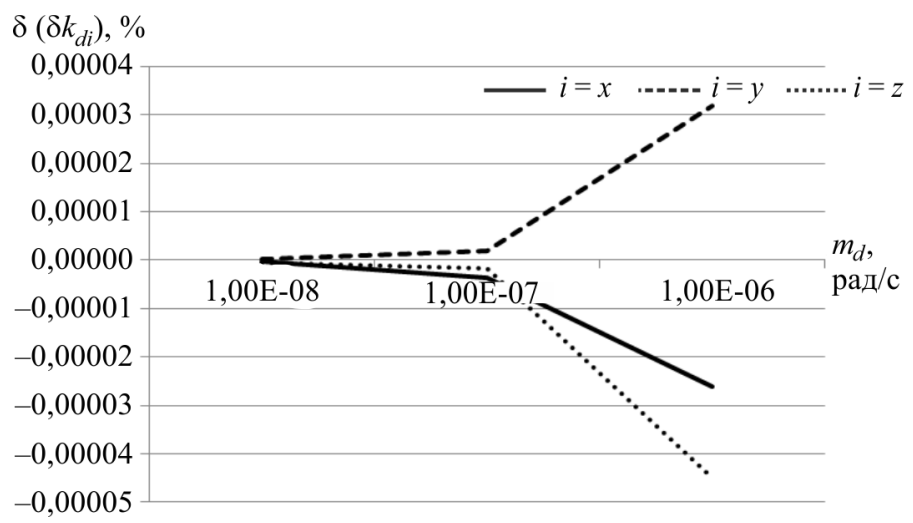

Рис. 2. График зависимости $\delta\left(\delta k_{d i}\right)=f\left(m_{d}\right)$

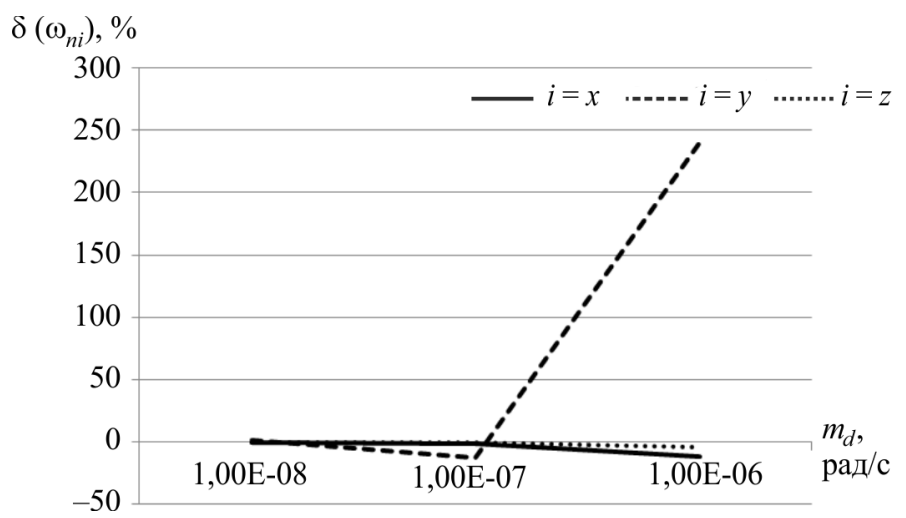

Рис. 3. График зависимости $\delta\left(\omega_{n i}\right)=f\left(m_{d}\right)$

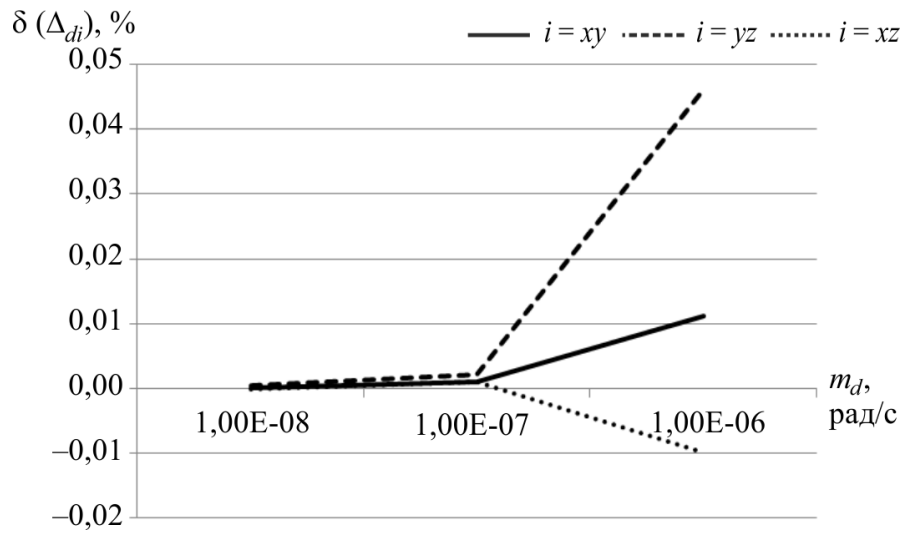

Рис. 4. График зависимости $\delta\left(v_{i j}\right)=f\left(m_{d}\right)$ 
Проведем анализ зависимостей ошибок калибровочных коэффициентов от уровня шумов волоконно-оптических гироскопов. Из графиков следует, что ошибки масштабных коэффициентов и углов перекосов (см. рис. 2, 4) практически не зависят от уровня шумов гироскопов. Ошибки смещения нулей $\omega_{n i}$ горизонтальных ВОГ (см. рис. 3 ) также мало зависят от уровня шумов. Ошибка смещения нуля вертикального гироскопа при достижении уровня шума до значений, выходящих за пределы дрейфов гироскопов навигационного класса, резко возрастает и достигает недопустимых значений. На всех графиках есть точка на оси абцисс, начиная с которой растут ошибки всех калибровочных коэффициентов. Это явление можно объяснить тем, что из-за роста $\omega_{n y}$ за счет перекрестных связей растут ошибки калибровочных коэффициентов. Рост смещения нуля $\omega_{n y}$ вызывается тем, что сигнал вертикального волоконно-оптического гироскопа не проходит через шулеровский контур, который является фильтром для шумовой составляющей.

\section{5. Анализ результатов моделирования}

Анализируя результаты моделирования, можно сделать следующие выводы:

1. Предложенный метод идентификации инструментальных погрешностей волоконно-оптических гироскопов БИНС по ошибкам ее выходных сигналов является эффективным.

2. Точность идентификации инструментальных погрешностей волоконно-оптических гироскопов БИНС зависит от уровня шума.

3. Метод наиболее эффективен для БИНС с инерциальными измерителями навигационного класса.

4. Для повышения точности идентификации инструментальных ошибок волоконно-оптических гироскопов целесообразно использовать трехканальную БИНС с возможностью пропускать сигналы всех трех ВОГ через шулеровский контур.

\section{Список литературы}

1. Андреев А.Г., Ермаков В.С., Николаев С.Г., Колеватов А.П. Способ калибровки бесплатформенных инерциальных навигационных систем. - Патент РФ № 2406973. Приоритет 10.08.2010.

2. Николаев С.Г. Калибровка бесплатформенных инерциальных навигационных систем // Известия вузов. Сер.: Приборостроение.СПб.: Изд-во СПбГУ ИТМО, 2009. - Т. 52, № 7. - С. 50-55. 
3. D. Titterton and J. Weston. Strapdown Inertial Navigation Technology. - 2nd ed. - The Institution of Electrical Engineers, 2004. - 547 p.

4. Деревянкин А.В., Матасов А.И. Методика калибровки блока акселерометров при грубой информации о его угловом положении. М.: МГУ им. М.В. Ломоносова, 2006. - 69 с.

\section{References}

1. Andreev A.G., Ermakov V.S., Nikolaev S.G., Kolevatov A.P. Sposob kalibrovki besplatformennykh inertsial'nykh navigatsionnykh system [The method of calibration of strapdown inertial navigation systems]. Patent PU № 2406973. 10.08.2010.

2. Nikolaev S.G. Kalibrovka besplatformennykh inertsial'nykh navigatsionnykh system [Calibration of strapdown inertial navigation systems]. Izvestiia vuzov. Seriia "Priborostroenie", 2009, vol. 52, no. 7, pp. 50-55.

3. Titterton D., Weston J. Strapdown Inertial Navigation Technology. The Institution of Electrical Engineers, 2004, 547 p.

4. Dereviankin A.V., Matasov A.I. Metodika kalibrovki bloka akselerometrov pri gruboi informatsii o ego uglovom polozhenii [The method of calibration block accelerometers with rough information about the angular position]. Moscow: Moskovskii gosudarstvennyi universitet imeni M.V. Lomonosova, 2006, 69 p.

Получено 26.11.2014 